\title{
Protocol Amendment Grace Period
}

National Cancer Institute

\section{Source}

National Cancer Institute. Protocol Amendment Grace Period. NCI Thesaurus. Code C93635.

The period of time during which study sites can continue to use an existing study version before they are required to switch to the new study version without incurring penalties. 